\title{
Classificazione, diagnosi ed ICD-10. III - Le sindromi affettive
}

\author{
STYLIANOS NICOLAOU' e GIOVANNI de GIROLAMO² \\ 1 Servizio di Psicologia Medica, Istituto di Psichiatria, Università di Verona \\ 2 Division of Mental Health, World Health Organization, Geneva
}

\begin{abstract}
Riassunto. Scopo - Presentare un breve profilo storico sui termini nosografici e sui principali modelli di classificazione dei disturbi affettivi descrivendo le caratteristiche più importanti della loro classificazione secondo l'ICD-10, le principali novità introdotte in essa rispetto all'ICD-9 ed infine alcune similarità e differenze tra la classificazione ICD-10 ed il DSM-III-R. Risultati - Dall'analisi della struttura della classificazione di questi disturbi nell'ICD-10 emerge con chiarezza la difficoltà di arrivare ad una sistemazione nosografica esauriente sulla base delle conoscenze attuali. L'ICD-10, comunque, ha segnato un significativo progresso rispetto all'ICD-9, ed ha, al tempo stesso incorporato molti degli orientamenti riscontrabili nel DSM-III-R. Conclusioni - L'ICD-10 ha proposto un modello di classificazione che cerca di operare una sintesi tra tradizioni e scuole diverse, e di valorizzare nel contempo, per quanto possibile, $i$ risultati emersi dalle ricerche di queste ultime 2 decadi.
\end{abstract}

Parole chiave: sindromi affettive, diagnosi, ICD-10.

Summary. Objective - To discuss, after a description of the main models of classification of affective disorders, the main features of the ICD-10 classification of these disorders, the main differences between ICD-10 and ICD-9 and a comparison with DSM-III-R classification. Results - The analysis of the classification of these disorders as reported in ICD-10 shows that their nosographic sistematization is a complex task. In any case ICD-10 represents a significant improvement compared to ICD-9 and has included many of the guidelines of DSM-III-R. Conclusions - ICD-10 proposes a syntesis between different schools and traditions taking into account the results emerged by research in this field over the last twenty years.

Key words: affective disorders, diagnosis, ICD-10.

Ricevuto il 4.3.1993 - Revisione ricevuta il 10.5.1993 - Accettato il 17.5.1993.

\section{INTRODUZIONE}

I problemi inerenti alla classificazione ed alla diagnosi delle sindromi affettive rivestono una importanza particolare in psichiatria per due ragioni fondamentali: in primo luogo perché i disturbi affettivi costituiscono, da un punto di vista epidemiologico, il gruppo di disturbi psichici di più comune riscontro e di maggiore rilievo in termini di sanità pubblica (de Girolamo, 1993), e manifestano inoltre una tendenza all'aumento in termini di incidenza, al punto che Klerman (1979) ha chiamato questa l'età della melanconia; in secondo luogo perché la concettualizza-

Indirizzo per la corrispondenza: Dr. G. de Girolamo, Division of Mental Health, World Health Organization, 1211 Geneva 27, Switzerland.

Fax (+41) 22-791.0746. zione, in termini nosografici, di questi disturbi ha rappresentato storicamente uno dei capitoli più problematici per l'intera psichiatria.

In questo contributo saranno innanzitutto discussi i principali modelli di classificazione dei disturbi affettivi; quindi sarà delineata la struttura della classificazione di queste sindromi nell'ICD-10, ed infine tale classificazione sarà comparata con la classificazione del DSM-III-R, al fine di evidenziarne similarità e differenze.

\section{PRINCIPALI MODELLI CLASSIFICATORI DEI DISTURBI AFFETTIVI SINO ALL'ICD-10}

Nell'ambito delle intricate e spesso complesse vicende che da oltre un secolo a questa parte hanno 
avuto luogo nel campo della nosografia e tassonomia psichiatrica, quelle relative alla classificazione dei disturbi affettivi occupano un posto di assoluto rilievo: infatti, differenti modelli e proposte di classificazione di questi disturbi si sono succedute e spesso giustapposte, riflettendo differenti modelli teorici di riferimento e differenti opzioni teoriche (Kendell, 1976). Il problema è stato aggravato dal fatto che gli stessi termini hanno spesso assunto differenti significati in differenti schemi diagnostici, mentre termini differenti hanno talora avuto uguali significati in differenti modelli diagnostici (Klerman, 1980).

Questa intricata vicenda nosografica ha tratto la sua ragione centrale di essere dall'esigenza di separare, in maniera euristicamente valida, la nozione «di depressione come manifestazione di un disagio esistenziale dalla depressione intesa come malattia definita" (Saraceno et al., 1989). Intorno a questa esigenza fondamentale si sono venuti costruendo la maggior parte degli approcci classificatori che saranno ora descritti; nel far ciò ci si baserà in parte sulla chiara esposizione di Gelder et al. (1990).

\section{Classificazioni basate sull'eziologia}

I primi approcci classificatori da considerare sono quelli basati sulla (supposta) eziologia di questi disturbi: a loro volta questi modelli di classificazione possono essere ulteriormente suddivisi in classificazioni basate sulla dicotomia endogeno/reattivo e sulla dicotomia primario/secondario.

\section{La classificazione endogeno/reattivo}

Sulla base di questo schema, i disturbi depressivi sono stati suddivisi in endogeni e reattivi (talvolta chiamati nel passato esogeni). Nel primo caso venivano interpretati quali disturbi sorti sulla base di fattori di natura costituzionale, genetici o biochimici senza alcuna relazione con stressori ambientali; nel secondo caso invece questi disturbi venivano considerati come una reazione psichica a situazioni ambientali o interpersonali di stress, ed in particolare ad esperienze di perdita. Negli anni più recenti, la scuola di Newcastle ha particolarmente insistito su tale modalità di classificazione (Carney et al., 1965).

$\mathrm{Va}$ detto che già in passato tale modalità di classificazione è stata fortemente criticata (vedasi, ad esempio, Lewis, 1971). Ed infatti numerosi studi hanno messo in discussione un siffatto approccio categoriale, centrato sull'antinomia endogeno/reattivo: in particolare, ripetute indagini di tipo epidemiologico, condotte sia nella popolazione generale che nella medicina di base, hanno mostrato l'assenza di qualsiasi confine netto tra queste due forme di depressione e la presenza di una gradazione pressocché continua tra casi di differente gravità, tendendo in tal senso piuttosto a validare modelli di tipo dimensionale nella concettualizzazione del disturbo (Paykel \& Rowan, 1979; Brown \& Harris, 1989).

Tra le più recenti ed esaurienti ricerche a questo proposito va citato il Camberwell Collaborative Depression Study (Bebbington et al., 1988; Bebbington \& McGuffin, 1989). In questa indagine sono stati attentamente studiati 130 pazienti ( 76 donne e 54 uomini) che erano venuti in contatto con un servizio psichiatrico londinese e che avevano una diagnosi di depressione unipolare; sono stati inoltre esaminati i loro familiari. Il principale risultato emerso da questo studio, relativamente alla discussione in oggetto, è che sia nei casi di depressione categorizzabile come endogena che in quella definibile come reattiva era possibile rinvenire un numero molto più elevato di eventi stressanti e di difficoltà esistenziali nei mesi precedenti l'insorgenza dell'episodio depressivo, in confronto con la popolazione generale; non era quindi possibile differenziare $\mathrm{i}$ due supposti tipi di depressione rispetto alla presenza o meno di eventi stressanti. Pertanto, «i dati di questo studio non supportano una differenza tra depressione endogena e depressione nevrotica in rapporto alla presenza di avversità sul piano psicosociale, e la distribuzione temporale degli eventi [stressanti] è altamente indicativa di un loro ruolo causale in entrambi i tipi di depressione» (Bebbington et al., 1988). L'elevata presenza di eventi stressanti prima dell'episodio di depressione era relativa non solo ad eventi stressanti gravi, ma anche a quelli di minore gravità ed entità; vi era inoltre una differenza statisticamente significativa tra i due sessi rispetto al numero di eventi stressanti, con un numero di life events più elevato tra le donne. Tali risultati sono in sintonia con quelli ottenuti in altre ricerche, come viene sottolineato dagli stessi autori (Bebbington \& McGuffin, 1989). Inoltre, anche nel caso della familiarità non era possibile riscontrare una differenza tra i due tipi di depressione nella direzione che sarebbe stata prevista, ossia con un eccesso di casi di depressione nelle famiglie dei cosiddetti soggetti endogeni (McGuffin et al., 1988a; 1988b).

Sia I'ICD-10 che il DSM-III-R non fanno alcun riferimento alla dicotomia endogeno/reattivo, che è pertanto da considerarsi obsoleta. 


\section{La classificazione primario/secondario}

Questo tipo di classificazione è altrettanto basata su una differenziazione in termini di possibile eziologia, e fu introdotta a scopi di ricerca nel 1972 dalla scuola di St. Louis (Feighner et al., 1972), al fine di escludere, negli studi sulla depressione, pazienti il cui disturbo poteva essere considerato secondario ad altri disturbi psichiatrici non affettivi (quali schizofrenia, alcolismo, disturbi d'ansia), o anche alla presenza di malattie somatiche, o infine alla assunzione di farmaci.

Nell'ICD-10 tale distinzione trova un parziale fondamento, dal momento che da una parte è prevista la possibilità di fare diagnosi di sindromi affettive propriamente dette o "primarie», e dall'altra possono essere diagnosticate patologie affettive cosidette "secondarie» come ad esempio le sindromi affettive organiche, le sindromi psicotiche con sintomi prevelentemente affettivi, ecc.

\section{Classificazioni basate sui sintomi psicotico/nevrotico}

La presenza di differenti quadri sintomatologici riscontrabili negli episodi depressivi ha condotto alla differenziazione tra depressioni di tipo psicotico (tra le quali veniva inclusa, anche la melanconia che viene descritta nel DSM-III-R come una depressione tipicamente grave con caratteristiche endogene, o depressione maggiore) e di tipo nevrotico. Va tuttavia detto, come sottolineato da Gelder et al. (1990), che l'uso non uniforme del termine psicotica ha condotto a differenti interpretazioni della stessa nozione di depressione psicotica: cosi, per alcuni questa forma di disturbo affettivo è divenuta sinonimo di depressione contrassegnata da sintomi quali allucinazioni e deliri, in particolare deliri di colpa e rovina, mentre da altri la nozione di depressione psicotica è stata soprattutto applicata ai casi caratterizzati da una rilevante sintomatologia clinica quali perdita di peso, disturbi del sonno, fluttuazioni diurne nell'intensità della sintomatologia, ecc. In generale, le ricerche mirate a validare tale tipo di differenziazione hanno fornito risultati talvolta contrastanti, che non consentono nè di affermare con sicurezza nè di rigettare l'ipotesi relativa all'esistenza di queste due forme di depressione; in termini prognostici, questa specificazione diagnostica consente solo di predire con qualche accuratezza la natura dei sintomi in future ricadute, mentre non consente di predire il rischio stesso di recidive (Jablensky, 1987). Quindi il termine «de- pressione psicotica», indipententemente dalla presenza o meno di sintomi psicotici, viene utilizzato nella letteratura con significati diversi per descrivere forme di depressione di notevole gravità e con aspetti psicopatologici di tipo endogeno (Maggini et al., 1993)

I termini psicotico e nevrotico erano anche utilizzati in riferimento ad ipotesi di tipo eziologico (alla depressione psicotica veniva attribuita un'origine costituzionale, genetica o biochimica, mentre l'origine della depressione nevrotica veniva ascritta all'ambiente ed a fattori psicosociali e di personalità).

Sempre con riferimento alle sindromi affettive, va detto che l'uso del termine psicosi aveva nell'ICD-9 un ruolo determinante per la denominazione delle varie categorie, mentre sia nell'ICD-9-CM che nel DSM-III-R tale termine è stato del tutto sostituito dal termine disturbo.

Nel capitolo dell'ICD-10 relativo alle sindromi affettive è prevista, sulla base di un criterio puramente descrittivo e nel rifiuto esplicito di qualsiasi riferimento di tipo eziologico, la possibilità di identificare specifiche categorie di disturbi affettivi - sia nel caso della mania sia nel caso della depressione caratterizzate dalla presenza di sintomi psicotici, rappresentati essenzialmente da deliri ed allucinazioni.

Tuttavia, la presenza di aspetti psicotici in un paziente affetto da una sindrome affettiva, ancorché importante da un punto di vista clinico e terapeutico, ha un significato nosografico incerto ai fini della classificazione delle sindromi affettive.

\section{Classificazioni basate sul tipo di decorso}

Nella sua tradizionale classificazione, Kraepelin elaborò il concetto di psicosi maniaco-depressiva sulla base di un criterio relativo al decorso: egli infatti riteneva che il decorso del disturbo fosse sostanzialmente unico e pertanto classificò in questa categoria sia le forme depressive ricorrenti che le forme con episodi maniacali e depressivi. Tale impostazione, che fu largamente accettata in campo psichiatrico, $\mathrm{fu}$ quindi oggetto di revisione da parte di Leonhard (1959), il quale suggerì di differenziare tre gruppi di pazienti con disturbi affettivi: quelli con depressione unipolare, con mania unipolare e con un disturbo bipolare. Il gruppo della mania unipolare è stato successivamente fatto rientrare nel gruppo bipolare, sulla base dei dati che mostrano come pressocché tutti coloro che soffrono di più di un episodio di mania finiscano poi per evidenziare anche un episodio di 
depressione. I disturbi bipolari ed unipolari, pertanto, sono stati differenziati sia sul piano clinico che per quanto riguarda l'età di esordio, il decorso, il numero degli episodi e l'incidenza nei due sessi (il disturbo unipolare è più frequente nelle femmine, mentre nei bipolari il rapporto è 1:1) (Paykel, 1989). Va sottolineato che solo una piccola frazione di tutti gli episodi depressivi, pari a circa il $10 \%$, sono bipolari (Paykel, 1989). Sono state riportate in letteratura differenze anche genetiche, di personalità e biologiche tra le due forme (Allen, 1976). Inoltre i bipolari rispondono meglio al litio (Schou, 1976). Il gruppo delle depressioni unipolari (termine che è stato spesso considerato equivalente a quello di depressione maggiore) è comunque ancora più eterogeneo rispetto alle forme bipolari (Schatzberg et al., 1982): sono riconoscibili forme ad esordio precoce e tardivo.

Tale impostazione è rimasta invariata nelle varie revisioni dell'ICD, sino all'ICD-9 ed all'ICD-9-CM, dove tra bipolarità ed unipolarità i confini sono divenuti più marcati. Anche il DSM-III ha differenziato i disturbi affettivi in bipolari ed in depressione maggiore. La validità di tale suddivisione dei disturbi affettivi è stata di recente confermata da Blacker \& Tsuang (1992) sulla base di una accurata ed estesa revisione della letteratura.

Nonostante anche la differenziazione tra forme bipolari ed unipolari conduca a qualche sovrapposizione tra pazienti appartenenti all'uno o all'altro gruppo, essa è forse la più utile tra quelle sinora discusse, soprattutto perché essa consente di fare delle precise predizioni in termini di risposta al trattamento (soprattutto per quanto riguarda il trattamento profilattico con litio).

La differenziazione tra diverse forme di depressione in rapporto al decorso è stata ripresa nell'ICD-10, che contiene una categoria specifica per la sindrome affettiva bipolare, che viene contrapposta agli episodi singoli di tipo maniacale o depressivo.

Oltre agli approcci classificatori discussi sino a questo punto, se ne possono individuare altri, come ad esempio quello di Lewis (1971), secondo cui la malattia può manifestarsi secondo due varianti, ossia severa/acuta e moderata/cronica, o quello proposto da Paykel (1971), che ha riconosciuto l'esistenza di quattro gruppi di pazienti depressi, da lui definiti psicotici, ansiosi, ostili ed infine con disturbi della personalità; questi, ed altri modelli di categorizzazione dei disturbi depressivi non hanno tuttavia assunto un rilievo analogo ai modelli discussi in precedenza.
Si può quindi concludere questa breve discussione dei principali approcci classificatori sottolineando che essi sono stati contrassegnati da una continua tendenza ad una «organizzazione bimodale» della fenomenologia depressiva (Saraceno et al., 1989), corrispondente alla già citata differenziazione tra depressione come forma di disagio esistenziale e depressione come malattia. Da questa esigenza fondamentale sono derivati i modelli sopra presentati, la cui validità, come si è visto, ha retto solo in parte ad accurati tentativi di verifica scientifica.

\section{LE SINDROMI AFFETTIVE NELL'ICD-10}

Nel proporre una classificazione delle sindromi affettive; il testo dell' ICD-10 riconosce con chiarezza la difficoltà di arrivare ad una sistematizzazione nosografica esauriente di tali sindromi sulla base delle conoscenze attuali: viene infatti apertamente riconosciuto che «gli psichiatri continueranno ad essere in disaccordo circa la classificazione della sindromi affettive, finché non saranno scoperti metodi per suddividere le sindromi cliniche basati almeno in parte su misure fisiologiche o biochimiche, anziché, come al momento, soltanto su descrizioni cliniche di emozioni o comportamenti» (WHO, 1992, p. 14). Viene quindi esplicitamente riconosciuta la difficoltà di operare una suddivisione sulla base di criteri eminentemente descrittivi ed in assenza di criteri di validazione esterna, quali ad esempio la presenza di markers fisiologici o biochimici.

Sulla base dell'esteso processo di consultazione svoltosi durante i field trials, è stato quindi deciso di differenziare vari gradi di depressione, di separare gli episodi sulla base della gravità e della loro ricorrenza o meno, di specificare la presenza o l'assenza di sintomi psicotici quali deliri ed allucinazioni congrui con l'umore del soggetto, ed infine di separare la mania dalla ipomania.

La tabella I riporta, in maniera semplificata, le principali categorie di sindromi affettive secondo 1'ICD-10; va sottolineato che i criteri diagnostici che hanno informato la classificazione di questi disturbi nell'ICD-10 sono stati rappresentati soprattutto dalla "loro facile applicabilità nella pratica clinica» (WHO, 1992, p. 106): pertanto gli episodi bipolari e depressivi ricorrenti sono stati distinti da quelli singoli, ed è stata attribuita particolare importanza alla gravità di ciascun episodio, per le implicazioni di na- 
Tabella I. - Le sindromi affettive nell'ICD-10 (F30-F39).

F30 Episodio maniacale

- F30.0 Ipomania

- F30.1 Mania senza sintomi psicotici

- F30.2 Mania con sintomi psicotici

- F30.8 Altro

- F30.9 Non specificato

F31 Sindrome affettiva bipolare

- F31.0 Attuale episodio ipomaniacale

- F31.1 Attuale episodio maniacale senza sintomi psicotici

- F31.2 Attuale episodio maniacale con sintomi psicotici

- F31.3 Attuale episodio depressivo lieve o di media gravità

- F31.4 Attuale episodio depressivo grave senza sintomi psicotici

- F31.5 Attuale episodio depressivo grave con sintomi psicotici

- F31.6 Attuale episodio misto

- F31.7 Attualmente in remissione

- F31.8 Altra

- F31.9 Non specificata

F32 Episodio depressivo

- F32.0 Episodio depressivo lieve

- F32.1 Episodio depressivo di media gravità

- F32.2 Episodio depressivo grave senza sintomi psicotici

- F32.3 Episodio depressivo grave con sintomi psicotici

- F32.8 Altro

- F32.9 Non specificato

F33 Sindrome depressiva ricorrente

- F33.0 Episodio attuale lieve

- F33.1 Episodio attuale di media gravità

- F33.2 Episodio attuale grave senza sintomi psicotici

- F33.3 Episodio attuale grave con sintomi psicotici

- F33.4 Attualmente in remissione

- F33.8 Altra

- F33.9 Non specificata

F34 Sindromi affettive persistenti

- F34.0 Ciclotimia

- F34.1 Distimia

- F34.8 Altra

- F34.9 Non specificata

F38 Altre sindromi affettive

- F38.0 Episodi affettivi singoli di altro tipo

- F38.1 Sindromi affettive ricorrenti di altro tipo

- F38.8 Altre specificate

F39 Sindrome affettiva non specificata

tura assistenziale che essa riveste; sono stati pertanto riconosciuti tre livelli di gravità, lieve, medio e grave. Per le forme gravi è prevista l'ulteriore suddivisione in forme con aspetti psicotici e non.

Per quanto riguarda la ricorrenza dei disturbi affettivi, si è tenuto conto del fatto che alcuni pazienti possono presentare un solo episodio mentre altri presentano episodi multipli; una distinzione quindi è stata fatta tra episodi bipolari, episodi multipli ed episodi singoli.

In generale, si può affermare che la sezione dell'ICD-10 sui disturbi affettivi ha segnato un significativo progresso rispetto all'ICD-9, ed ha, al tempo stesso, incorporato molti degli orientamenti riscontrabili nel DSM-III-R. Verrano ora descritti separatamente i principali gruppi di disturbi affettivi secondo l'ICD-10.

\section{Episodio maniacale}

Gli elementi clinici essenziali (criteri) per fare diagnosi di episodio maniacale sono i seguenti: l'esaltazione del tono dell'umore e l'incremento ed accelerazione dell'attività fisica e mentale (di tipo sessuale, lavorativo e sociale). Questi sintomi possono subire oscillazioni lievi (ipomania) oppure notevoli come nel caso della mania con eccitamento psicomotorio. Altri sintomi che spesso caratterizzano il quadro clinico sono l'eccessiva autostima (idee di grandezza, eccessivo ottimismo), aumentato flusso del linguaggio fino alla fuga delle idee, diminuito bisogno di sonno, distraibilità, ecc. Il set dei sintomi appena descritti soddisfa i criteri per fare diagnosi di mania senza sintomi psicotici (F30.1) o di sindrome affettiva bipolare senza aspetti psicotici (F31.1). Nel caso, invece, in cui siano presenti sintomi psicotici (vedi categorie F30.2 o F31.2) tali sintomi consistono in deliri ed allucinazioni, iperattività che può arrivare all'eccitamento e a comportamenti aggressivi e violenti, fuga delle idee con linguaggio a volte incomprensibile e infine idee di grandezza e convinzioni deliranti con temi, molto spesso, di grandiosità e religiosità. L'intensa compromissione sul piano lavorativo e sociale può rendere necessaria l'ospedalizzazione.

Secondo l'ICD-10, la diagnosi di episodio maniacale singolo ( $\mathrm{F} 30$ ) va posta solo se tale episodio si manifesta in una persona senza alcuna anamnesi di disturbi affettivi precedenti, sia di tipo depressivo che maniacale; qualora tale anamnesi sia invece positiva, le Direttive Diagnostiche suggeriscono di fare diagnosi di sindrome affettiva bipolare. Sono pertanto indicati tre livelli di gravità, che hanno naturalmente in comune l'esaltazione del tono dell'umore.

Il disturbo, nella sua forma ipomaniacale (F30.0, F31.0), si presenta con una sintomatologia simile ma meno accentuata e grave di quella maniacale, e non vi è una marcata compromissione della capacità lavorativa o dell'adattamento psicosociale. 


\section{Sindrome affettiva bipolare}

Mentre nell'ICD-9 la psicosi maniaco-depressiva includeva non solo i veri pazienti bipolari, ma anche quelli che soffrivano soltanto di depressione, l'ICD-10 ha collocato quest'ultimo gruppo di pazienti nelle categorie dei disturbi depressivi con episodi singoli o in quelli ricorrenti.

Il concetto di bipolarità, o meglio la distinzione unipolare-bipolare, costituisce un criterio diagnostico molto importante sia nell'ICD-10 che nel DSMIII-R sostenuta in letteratura dal riscontro di differenze tra le due forme (unipolare e bipolare) per quanto riguarda le caratteristiche sociodemografiche, familiari, biologiche e di risposta al trattamento delle persone affette dall'uno o dall'altro disturbo (Maggini et al., 1993).

La sindrome affettiva bipolare è caratterizzata dal ripetersi di episodi di alterazione del tono dell'umore (in pratica almeno due) di tipo sia depressivo che maniacale. Il testo dell'ICD-10 afferma esplicitamente che tale categoria corrisponde a quella classica della psicosi maniaco-depressiva (ICD-9), ma con criteri diagnostici e confini categoriali più specifici e quindi più differenziati.

Tra i criteri in base ai quali viene definita la bipolarità è fondamentale, per l'ICD-10, il tipo degli episodi precedenti. Nel caso in cui l'attuale episodio sia di tipo ipomaniacale o maniacale, il disturbo si considera bipolare anche se in passato il soggetto ha avuto un'altro episodio dello stesso tipo, ossia ipomaniacale o maniacale. Al contrario che per gli episodi depressivi ricorrenti non viene quindi prevista la possibilità di diagnosticare episodi maniacali ricorrenti, in quanto si assume che coloro che hanno due o più episodi di questo tipo finiranno comunque con l'evidenziare in seguito episodi di natura depressiva.

L'ulteriore suddivisione diagnostica viene operata sulla base dell'episodio in atto al momento della valutazione diagnostica: pertanto, viene distinto un attuale episodio maniacale o ipomaniacale, $o$, al contrario, depressivo di differente gravità e con o senza sintomi psicotici.

\section{Episodio depressivo}

L'ICD-10 riconosce tre sintomi distintivi per ciascun episodio depressivo, rappresentati dalla depressione del tono dell'umore, dalla perdita degli interessi e della capacità di provare piacere ed infine dalla diminuzione dell'energia con accresciuta affaticabili- tà e diminuita attività. Accanto a questi, vengono descritti una serie di altri sintomi, tra cui la riduzione dell'attenzione e della concentrazione, la compromissione dell'autostima e della fiducia di sè, la presenza di idee di colpa e di inutilità, la visione pessimistica del futuro, l'insonnia o l'ipersonnia, e la diminuzione dell'appetito. Alcuni soggetti manifestano tuttavia una spiccata sintomatologia sul piano somatico, con risveglio mattutino precoce, fluttuazioni diurne della sintomatologia, rallentamento psicomotorio, marcata anoressia, diminuzione della libido e perdita di peso; i sintomi descritti soddisfano i criteri per fare diagnosi di episodio depressivo singolo (F32), di sindrome depressiva ricorrente (F33) e di sindrome affettiva bipolare con attuale episodio depressivo (F31.3, F31.4) senza aspetti psicotici. In ogni caso, per poter fare diagnosi di episodio depressivo si richiede di solito la presenza del quadro sintomatologico per almeno due settimane.

I confini tra le varie forme di episodio depressivo vengono definiti in base al numero ed all'entità dei sintomi, e quindi in rapporto alla conseguente disorganizzazione e/o menomazione del funzionamento sociale e lavorativo. In questo modo viene operata una suddivisione in varie sottocategorie sia rispetto alla gravità che alla presenza o meno di sintomi psicotici. In particolare per quanto riguarda le depressioni gravi l'ICD-10, come anche il DSM-III-R (il quale a sua volta prevede la diagnosi dell'episodio depressivo maggiore di tipo melanconico, cronico o meno) descrive il quadro clinico delle depressioni con sintomi psicotici (F32.3, F33.3, F31.5) come contrassegnato da una profonda sofferenza del soggetto, spesso legata ai deliri di rovina e ad allucinazioni (voci diffamatorie e accusatorie). Tali quadri clinici vengono suddivisi ulteriormente in due sottoclassi, rappresentate rispettivamente dal sottogruppo con manifestazioni psicotiche congrue all'umore (con deliri ed allucinazioni il cui contenuto ripropone le tematiche di colpa, rovina e ipocondria) e dal sottogruppo con aspetti psicotici incongrui all'umore (con sintomi paranoidei o sintomi di primo rango di Schneider).

L'episodio depressivo singolo è un episodio che si manifesta per la prima volta in una persona che non ha avuto nessuna sindrome affettiva precedentemente.

\section{Sindrome depressiva ricorrente}

Vanno qui incluse le condizioni psicopatologiche caratterizzate da ripetuti episodi di depressione, che 
soddisfano i criteri clinici dell'episodio depressivo di grado lieve, medio o grave, ma all'anamnesi episodi maniacali o ipomaniacali precedenti. Vanno inclusi in questa categoria diagnostica anche alcuni lievi episodi di tipo ipomaniacale insorgenti dopo la risoluzione dell'episodio depressivo (talvolta apparentemente precipitati dal trattamento antidepressivo). Per tale motivo tali episodi non devono essere diagnosticati come sindrome bipolare in quanto la sintomatologia ipomaniacale non è di tipo primario. Non va peraltro eslcusa la possibilità che alcuni pazienti con diagnosi di sindrome depressiva ricorrente possano andare successivamente incontro ad un episodio maniacale od ipomaniacale vero e proprio che soddisfa a questo punto i criteri della sindrome affettiva bipolare. Vanno inclusi tra le sindromi depressive ricorrenti anche quei casi che hanno presentato ripetuti episodi depressivi ed in cui la remissione tra i vari episodi non è stata completa). Comunque, nella maggior parte dei pazienti con episodi depressivi ricorrenti la remissione è completa. La durata degli episodi singoli può variare da un minimo di due settimane ad un massimo di dodici mesi con una durata media di circa quattro-sei mesi.

\section{Sindromi affettive persistenti}

L'ICD-10 ha riconosciuto l'esistenza di una categoria di sindromi affettive persistenti, tra le quali ha situato la distimia e la ciclotimia. Si tratta di quadri clinici contrassegnati da un disturbo persistente e di solito fluttuante dell'umore, di solito mai di gravità tale da poter tuttavia essere diagnosticati come ipomaniacali o di depressione anche lieve. La loro durata protratta, estesa talora all'intero arco biologico della vita adulta del soggetto, fa sì che essi possano essere causa di notevole sofferenza e disabilità.

Nella ciclotimia (F34.0) prevale l'instabilità del tono dell'umore con numerose fasi, più o meno brevi di depressione o di esaltazione dell'umore stesso; tali fasi sono spesso così lievi da passare clinicamente inosservate (tra le fasi di alterazione dell'umore vi possono essere periodi intervallari liberi da sintomi di durata non superiore ai due mesi). L'esordio può avvenire nell'adolescenza (precoce) o all'inizio della vita adulta (tardivo). Nel primo caso (esordio precoce) l'esordio è insidioso ed il quadro clinico è caratterizzato da problemi comportamentali (continue oscillazioni dell'umore, problematiche relazionali, ecc.) che evocano aspetti o tratti personologici (infatti a volte è necessario differenziare la ciclotimia dal disturbo borderline di personalità). Per fare diagnosi viene richiesta una prolungata osservazione del soggetto, o una dettagliata anamnesi. Nell'ICD-10 la ciclotimia è considerata come una sindrome ed entità nosografica autonoma anche rispetto alla sindrome affettiva bipolare, nonostante che nella pratica clinica i confini tra ciclotimia, sindrome affettiva bipolare (di lieve gravità) e bipolare non specificata non siano di fatto facilmente definibili.

La distimia (F34.1) è parimenti considerata un'entità nosografica separata. Questo disturbo aveva già assunto una notevole importanza nel DSM-III ove per la prima volta era stato classificato autonomamente (si parlava infatti di disturbo distimico) ed aveva sostituto la diagnosi di personalità depressiva e di nevrosi depressiva utilizzate entrambe per molti anni in altri sistemi diagnostici in psichiatria (l'ICD-9 prevedeva nell'ambito dei disturbi nevrotici, una specifica categoria rappresentata dalla nevrosi depressiva e tra i disturbi di personalità quella di personalità depressiva). La diagnosi di distimia viene pertanto posta qualora sia presente un quadro di depressione cronica del tono dell'umore con una perdurante sensazione di stanchezza e di inadeguatezza, che tuttavia non soddisfa $\mathrm{i}$ criteri diagnostici propri della sindrome depressiva ricorrente di gravità lieve o media, per quanto riguarda l'entità o la durata dei singoli episodi. L'alterazione dell'umore è persistente o intervallata da brevi periodi di umore relativamente normale. L'esordio può essere precoce (prima dei 21 anni) o tardivo (dopo i 21 anni). Secondo alcuni autori (Keller, 1989) i distimici ad esordio precoce, a differenza di quelli ad esordio tardivo, manifestano più frequentemente disturbi di personalità, una maggiore familiarità di disturbi affettivi ed una più marcata compromissione sul piano psicosociale. Come si è visto nel caso della ciclotimia anche la distimia deve essere spesso differenziata dai disturbi di personalità in cui prevalgono segni psicopatologici molto simili ai sintomi affettivi e somatici tipici della distimia.

\section{Sindrome mista ansioso-depressiva}

Per quanto riguarda la sindrome mista ansiosodepressiva (F41.2) secondo l'ICD-10, essa non è stata collocata nella sezione relativa alle sindromi affettive, ma è stata invece inserita nel capitolo delle sindromi nevrotiche, legate a stress e somatoformi. Va ricordato che il DSM-III-R non prevede la diagnosi di un quadro misto ansioso-depressivo come entità 
nosografica separata. Si tratta, invece, di un quadro di frequente riscontro sia nei servizi psichiatrici che nei reparti medici ospedalieri e soprattutto nel contesto della medicina generale; esso è caratterizzato dalla presenza simultanea di sintomi d'ansia e depressivi che non sono però di entità tale da poter giustificare una diagnosi separata ed indipendente dei due disturbi. Tali sintomi hanno spesso un andamento variabile sia dal punto di vista temporale che della espressività clinica, e sono associati alla presenza fluttuante di sintomi vegetativi (gastrointestinali, cardiaci, tremori, ecc.). Tali disturbi sono spesso fonte di notevole sofferenza per il paziente e sono associati a significative disabilità sul piano psicosociale, per cui il loro adeguato riconoscimento e trattamento riveste grande importanza. Quando i sintomi sono correlati ad un significativo cambiamento di vita $o$ ad un evento stressante (la presenza di una malattia somatica, un lutto, una separazione ecc.) viene fatta diagnosi di reazione mista ansioso-depressiva (F43.22), che è una sottocategoria delle sindromi da disadattamento (F43.2).

\section{ICD-10, DSM-III-R E DSM-IV: SIMILARITÀ E DIFFERENZE NOSOGRAFICHE E DIAGNOSTICHE}

La tabella II riporta in maniera schematizzata, la classificazione dei disturbi affettivi secondo l'ICD-10, il DSM-III-R ed il DSM-IV (APA, 1993). Nella tabella le principali categorie incluse nei rispettivi sistemi classificatori sono indicate in maiuscolo, mentre le sottocategorie sono in minuscolo. Va detto che il sistema americano prevede un codice aggiuntivo al fine di specificare il livello di gravità secondo un totale di sette gradazioni (lieve, media gravità, grave con sintomi psicotici, grave senza sintomi psicotici, in remissione parziale, in remissione piena, non specificato).

Come si può vedere, l'ICD-10 presenta ampie similarità con la classificazione dei disturbi affettivi proposta sia dal DSM-III-R che dal DSM-IV (APA, 1993): in particolare i tre sistemi contengono categorie differenziate per gli episodi singoli così come per gli episodi ricorrenti di disturbo. In secondo luogo, $i$ tre sistemi prevedono la possibilità di disturbi dell'umore lievi ma persistenti, quali la ciclotimia e la distimia. Tuttavia, il DSM-IV ha operato una più minuziosa classificazione dei disturbi bipolari rispetto a quanto aveva fatto il DSM-III-R: anzitutto i disturbi bipolari vengono suddivisi in due classi, e la seconda classe viene meglio specificata come «Episodi ricorrenti di depressione maggiore con ipomania»; nella prima classe di disturbi bipolari vengono proposte due nuove sottocategorie (episodio maniacale singolo e disturbo bipolare con episodio recente di tipo ipomaniacale); infine viene inclusa una nuova categoria di «Disturbo affettivo dovuto ad una condizione di interesse medico generale».

Per quanto riguarda invece le differenze tra i due sistemi, va detto che l'ICD-10 contiene una categoria aggiuntiva rispetto al DSM-III-R, rappresentata dalle altre sindromi affettive (F38), a sua volta suddivisa in tre sottocategorie; la sindrome affettiva non specificata (F39) invece corrisponde solo in parte alla categoria dei disturbi depressivi non altrimenti specificati del DSM-III-R.Inoltre, i criteri diagnostici per la distimia indicati nel DSM-III-R sono piuttosto differenti da quelli contenuti nell'ICD-10: il Manuale americano ne parla infatti come di un disturbo cronico dell'umore, che si prolunga incessantemente per almeno due anni, associato a cambiamenti nell'appetito, nella stima di sè, nel livello di energia, nella capacità di concentrazione, ecc. L'ICD-10 prevede invece l'esistenza di periodi intervallari di durata variabile, durante i quali il soggetto si descrive in buona salute, e non precisa inoltre un periodo minimo per fare diagnosi.

Un'altra differenza tra i due sistemi diagnostici riguarda i criteri sulla gravità, sul numero e sulla durata dei sintomi. Nel differenziare diversi quadri nell'ambito dello stesso disturbo depressivo, l'ICD-10 attribuisce maggior importanza al livello di gravità dei sintomi (lieve, moderato, grave), mentre il DSMIII-R dà maggior peso al numero dei sintomi presenti. Infine, per quanto riguarda la durata dei sintomi o del quadro clinico, l'ICD-10, a differenza del DSM-III-R, prevede un'ulteriore sottocategoria, la sindrome depressiva breve ricorrente (F38.10) che è collocata tra le sindromi affettive ricorrenti di altro tipo (F38.1). Tale sindrome fa riferimento ad episodi ripetuti di depressione, che soddisfano $i$ criteri clinici per un episodio depressivo, ma la cui durata è inferiore alle due settimane.

Nel complesso, sembra giustificato asserire che i due sistemi classificatori mostrano un'elevato livello di concordanza sia rispetto alla terminologia impiegata che rispetto alle categorie diagnostiche. Tuttavia, Mellsop et al., (1991) hanno espresso un'opinione prudente al riguardo, sostenendo che, mentre vi è una elevata affidabilità per quanto riguarda le categorie diagnostiche maggiori, la situazione appare più problematica per quanto riguarda le sottoclassi. 
Tabella II. - Prospetto riassuntivo dei disturbi affettivi nell'ICD-10, nel DSM-III-R e nel DSM-IV.

\begin{tabular}{|c|c|c|}
\hline ICD-10 & DSM-III-R & DSM-IV \\
\hline & Disturbo bipolare & Disturbo bipolare I \\
\hline Episodio maniacale & & Episodio maniacale singolo \\
\hline \multicolumn{3}{|l|}{ Ipomania } \\
\hline \multicolumn{3}{|l|}{ Mania con sintomi psicotici } \\
\hline \multicolumn{3}{|l|}{ Sindrome affettiva bipolare } \\
\hline Attuale episodio ipomaniacale & & Episodio recente di tipo ipomaniacale \\
\hline Attuale episodio maniacale & Maniacale & Episodio recente di tipo maniacale \\
\hline Attuale episodio depressivo & Depressivo & Episodio recente di tipo depressivo \\
\hline Attuale episodio misto & Misto & Episodio recente di tipo misto \\
\hline \multirow[t]{2}{*}{ Non specificato } & Disturbo bipolare non altrimenti specificato & Disturbo bipolare non altrimenti specificato \\
\hline & & $\begin{array}{l}\text { Disturbo bipolare II (episodi di depressio- } \\
\text { ne maggiore ricorrenti con ipomania) }\end{array}$ \\
\hline $\begin{array}{l}\text { Episodio depressivo } \\
\text { (lieve, di media gravità, grave con o sen- } \\
\text { za sintomi psicotici }\end{array}$ & $\begin{array}{l}\text { Disturbi depressivi: } \\
\text { depressione maggiore, episodio singolo }\end{array}$ & $\begin{array}{l}\text { Disturbi depressivi: } \\
\text { depressione maggiore, episodio singolo }\end{array}$ \\
\hline $\begin{array}{l}\text { Sindrome depressiva ricorrente } \\
\text { (attuale episodio lieve, di media gravità, } \\
\text { grave senza o con sintomi psicotici, } \\
\text { attualmente in remissione) }\end{array}$ & $\begin{array}{l}\text { Disturbi depressivi } \\
\text { Ricorrenti }\end{array}$ & $\begin{array}{l}\text { Disturbi depressivi: } \\
\text { Ricorrenti }\end{array}$ \\
\hline \multicolumn{3}{|l|}{ Sindromi affettive } \\
\hline Ciclotimia & Ciclotimia & Disturbo ciclotimico \\
\hline Distimia & $\begin{array}{l}\text { Distimia (o nevrosi depressiva) } \\
\text { (primaria o secondaria, ad insorgenza pre- } \\
\text { coce o tardiva }\end{array}$ & Disturbo distimico \\
\hline Altre sindromi affettive & Disturbi depressivi non altrimenti specificati & $\begin{array}{l}\text { Disturbi depressivi non altrimenti specificati } \\
\text { Disturbo affettivo dovuto ad una condi- } \\
\text { zione di interesse medico generale }\end{array}$ \\
\hline
\end{tabular}

\section{RISULTATI DEI FIELD TRIALS DELL'ICD-10}

Nei field trials relativi all'ICD-10 (per una descrizione più generale si rinvia al primo di questi tre contributi), $\mathrm{i}$ disturbi inclusi in questo capitolo hanno ottenuto nel complesso un valore di Kappa pari a 0,77 (Sartorius et al, 1993). Tuttavia, allorquando veniva richiesto ai clinici di precisare meglio la diagnosi, differenziando tra i vari sottotipi di disturbi affettivi, i valori di Kappa differivano marcatamente: cosi, si andava da un massimo di 0,77 per la sindrome affettiva bipolare, attuale episodio maniacale, ad un minimo di 0,29 per la categoria della sindrome depressiva ricorrente, episodio attuale lieve. La diagnosi di episodio depressivo grave ha riportato un valore di Kappa pari a 0,67, mentre quella di distimia un valore basso, pari a 0,36 .

Per quanto riguarda le categorie con più basso valore di accordo diagnostico, nel caso della sindrome depressiva ricorrente esso sembrava da ascrivere ad un disaccordo tra i clinici a proposito della valutazione della ricorrenza, della gravità e della durata del disturbo, mentre nel caso della distimia esso era sostanzialmente dovuto alla difficoltà di differenzia- 
re tale disturbo dalla sindrome depressiva ricorrente e dalla sindrome mista ansioso-depressiva.

\section{CONCLUSIONI}

La classificazione dei disturbi affettivi rappresenta, da sempre, una delle sfide più ardue per l'intera ricerca psichiatrica; nel contempo, vista la diffusione di questi disturbi, e la crescente possibilità di individuare strategie selettive di trattamento, appare come un compito di estrema importanza. I precedenti modelli di classificazione, talora molto differenziati, riflettevano anche esigenze diverse dei clinici operanti in setting altrettanto diversi (servizi psichiatrici ospedalieri o ambulatoriali, ambulatori di medicina generale), che venivano in contatto con popolazioni di pazienti sofferenti di disturbi affettivi alquanto eterogenee, sia dal punto di vista della gravità che della fenomenologia clinica. L'ICD-10 ha proposto un modello di classificazione che cerca di operare una sintesi tra tradizioni e scuole diverse e di valorizzare nel contempo, per quanto possibile, i risultati emersi dalle ricerche di queste ultime due decadi. I prossimi anni diranno in che misura tale tentativo è stato incoronato da successo.

\section{BIBLIOGRAFIA}

Allen M.G. (1976). Twin studies of affective illness. Archives of General Psychiatry 33, 1476-1478.

American Psychiatric Association (1993). DSM-IV Draft Criteria. American Psychiatric Association: Washington.

Bebbington P. \& McGuffin P. (1989). Calamity, constitution and the origins of depression. In Depression: An Integrative Approach. (ed. K.R. Herbst and E.S. Paykel), pp.65-80. Oxford: Heinemann.

Bebbington P., Brugha T., MacCarthy B., Potter J., Sturt E., Wykes T., Katz R. \& McGuffin P. (1988). The Camberwell Collaborative Depression Study. I. Depressed probands: adversity and the form of depression. British Journal of Psychiatry 152, 754-765.

Blacker D. \& Tsuang M.T. (1992). Contested boundaries of bipolar disorder and the limits of categorical diagnosis in psychiatry. American Journal of Psychiatry 149, 1473-1483.

Brown G.W. \& Harris T.O. (1989). Depression. In Life Events and Illness (ed. G.W Brown and T.O. Harris), pp. 49-93. Guilford: New York.

Carney M.W.P., Roth M. \& Garside R.F. (1965). The diagnosis of depressive syndromes and the prediction of ECT response. British Journal of Psychiatry 111, 659-674.

de Girolamo G. (1993). Cross-cultural differences in depression. Focus in Depression 4, 28-38.

Feighner J.P., Robins E., Guze S.B., Woodruff R.A., Winokur G.\& Muoz R. (1972). Diagnostic criteria for use in psychiatric research. Archives of General Psychiatry 26, 57-63.

Gelder M., Gath D. \& Mayou R. (1990). Oxford Textbook of Psychiatry (2nd ed.). Oxford University Press: Oxford.

Jablensky A. (1987). Prediction of the course and outcome of depression. Psychological Medicine 17, 1-9.
Keller M.B. (1989). Current concepts in affective disorders. Journal of Clinical Psychiatry 50, 157-164.

Kendell R.E. (1976). The classification of depressions: a review of contemporary confusion. British Journal of Psychiatry 129, 15-28.

Klerman G.L. (1979). Is the age of melancholy? Psychology Today 12, 36-42.

Klerman G.L. (1980). Overview of affective disorders. In Comprehensive Textbook of Psychiatry, (ed. H.I. Kaplan, A.M. Freedman and B.J. Sadock.), pp. 1305-1319. Williams \& Wilkins: Baltimore.

Leonhard K. (1959). Aufteilung der Endogenen Psychosen. Akademie Verlag: Berlin.

Lewis A.J. (1971). Endogenous and exogenous: a useful dichotomy? Psychological Medicine 1, 191-196.

Maggini C., Lattanzi L. \& Sbrana A. (1993). Nosografia della depressione. In Psichiatria della Comunità (ed. F. Asioli, A. Ballerini e G.B. Ceroni), pp. 108-146. Bollati Boringhieri: Torino.

McGuffin P., Katz R., Aldrich J. \& Bebbington P. (1988a). The Camberwell Collaborative Depression Study. II. The investigation of family members. British Journal of Psychiatry 152, 766-774.

McGuffin P., Katz R. \& Bebbington P. (1988b). The Camberwell Collaborative Depression Study. III. Depression and the adversity in the relatives of depressed probands. British Journal of Psychiatry 152, 775-782.

Mellsop G.W., Thomas G.S., Ellis P.M., Purdie G., Crawshaw J. \& Mendis N. (1991). Reliability of the draft diagnostic criteria for research of ICD-10 in comparison with ICD-10 and DSM-III-R. Acta Psychiatrica Scandinavica 84, 332-335.

Paykel E.S. (1971). Classification of depressed patients: a cluster analysis derived grouping. British Journal of Psychiatry 118, 275-288.

Paykel E.S. (1989). The background: extent and nature of the disorder. In Depression: An Integrative Approach. (ed. K.R. Herbst and E.S. Paykel), pp. 3-20. Oxford: Heinemann.

Paykel E.S. \& Rowan P.R. (1979). Affective disorders. In Recent Advances in Clinical Psychiatry (ed. K. Granville-Grossman), pp. 37-90. Churchill Livingstone: Edinburgh.

Saraceno B., Bolongaro G., Cottatellucci T., Laviola F., Maranesi T., Sternai E. \& Tognoni G. (1989). La depressione. Ricerca e Pratica 30, 217-270.

Sartorius N., Kaelber C., Cooper J., Roper M., Rae D., Gulbinat W., Ustun B. \& Regier D. (1993). Progress towards achieving a common language in psychiatry: results from the field trial of the clinical guidelines accompanying the WHO classification of mental and behavioural disorders in the ICD-10. Archives of General Psychiatry 50, 115-124..

Schatzberg A.F., Orsulale P.J., Rosenbaum A.H., Maruta T., Kruger E.R., Cole O.J. \& Schildkraut J.J. (1982). Toward a biological classification of depressive disorders. II: Heterogeneity of unipolar depressions. American Journal of Psychiatry $139,471-475$.

Schou M. (1976). Prophylactic and maintenance therapy in recurrent affective disorders. In Depression: Behavioural, Biochemical, Diagnostic and Treatment Concepts (cd. D.M. Gallant and G.M. Simpson, pp. 309-334. Hallsted Press: New York.

World Health Organization (1992). International Classification of Diseases, 10th. Revision. WHO: Geneva. Ed. it.: Decima Revisione della Classificazione Internazionale delle Sindromi e dei Disturbi Psichici e Comportamentali. Descrizioni Cliniche e Direttive Diagnostiche (ed. D. Kemali, M. Maj, F. Catapano, S. Lobrace e L. Magliano). Masson: Milano. 